\title{
Luminal B tumors are the most frequent molecular subtype in breast cancer of North African women: an immunohistochemical profile study from Morocco
}

\author{
Hinde El Fatemi ${ }^{1 *}$, Sanae Chahbouni ${ }^{1}$, Sofia Jayi ${ }^{2}$, Kaoutar Moumna ${ }^{1}$, My Abdelilah Melhouf ${ }^{1}$, Abdelaziz Bannani ${ }^{2}$, \\ Omar Mesbahi ${ }^{3}$ and Afaf Amarti $^{1}$
}

\begin{abstract}
Background: Breast cancer may be classified into luminal A, luminal B, HER2+/ER-, basal-like and normal-like subtypes based on gene expression profiling or immunohistochemical (IHC) characteristics. The aim of our study is to show the molecular profile characteristic of breast cancer in the North African population of Morocco. This work showed preliminary results and correlations with clinicopathological and histological parameters. Three hundred and ninety primary breast carcinomas tumor tissues were immunostained for ER, PR, HER2, CK5/6, CK8/18 and Ki67 using paraffin tissue.

Methods: We reviewed 390 cases of breast cancer diagnosed on January 2008 to December 2011 at the Department of pathology, Hassan II teaching hospital, Fez, Morocco. Age, size tumor, metastatic profile, node involvement profile, histological type and immunohistochemical profile were studied.
\end{abstract}

Results: The average age was 46 years; our patients were diagnosed late with a high average tumor size. Luminal B subtype was more prevalent (41.8\%), followed by luminal A (30.5\%), basal-like (13, 6\%), Her2-overexpressing (9, 2\%), and unclassified subtype (4.9\%).

Conclusion: This study showed that molecular classification and biological profile may be different according to geographical distribution, to encourage further studies to know the genomic profile of tumors and the environment.

Virtual slide: http://www.diagnosticpathology.diagnomx.eu/vs/1675272504826544

Keywords: Breast cancer, Molecular classification, Luminal B subtype, Staging, Antibodies, Immunohistochemistry

\section{Background}

Breast cancer is a heterogeneous disease such that they may have different prognoses and respond to therapy differently despite similarities in histological types, grade and stage. Based on the presence or absence of expression of the estrogen receptor (ER), breast cancer is divided in two groups: ER+ and ER-. Genetic expression profile has identified two subtypes of the ER+ tumors: luminal A and luminal B. ER- tumors also include two subtypes, the HER2+ and the basal type. These subtypes

\footnotetext{
* Correspondence: hinde0012@hotmail.com

'Department of Pathology, Hassan II teaching hospital, Fez, Morocco

Full list of author information is available at the end of the article
}

differ in their biology and both demonstrate short disease-free periods after treatment and poorer outcome. In Morocco, it's the first cancer in women and is currently a major public health problem. The molecular classification in breast carcinomas is now based upon gene expression analysis using DNA microarrays and allows to identify at least five groups: luminal A, luminal B, HER2-overexpressing, basal-like and normal breastlike [1-3]. However, large-scale subtyping using gene expression profiling from formalin-fixed, paraffinembedded samples is not currently feasible and remains very expensive. Therefore, immunohistochemical markers have been used as surrogates tools for DNA

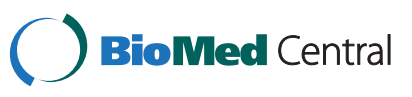

(c) 2012 EL FATEMI; licensee BioMed Central Ltd. This is an Open Access article distributed under the terms of the Creative Commons Attribution License (http://creativecommons.org/licenses/by/2.0), which permits unrestricted use, distribution, and reproduction in any medium, provided the original work is properly cited. 
microarray in subtyping breast cancer [4,5]. Several studies used routinely panels of immunohistochemical markers to classify breast cancers into subtypes similar to those previously defined using gene expression analyses [6]. The recent study was realized by Prat et al. [4]. They defined several immunohistochemical subtypes: (luminal A, Luminal B, HER2- enriched, basal-like) and a normal breast-like group that show significant differences in incidence, survival and response to therapy. Luminal A (ER positive (ER+) and/or PR positive (PR+), Her2 negative (Her2-)) with ki67 $<14 \%$, luminal B (ER + and/or $\mathrm{PR}+$ with ki67>14\%, Her2 positive or negative (Her2+/-), Her2+/ER - subtype (Her2+, ER-, PR-) and basal-like (ER-, PR-, Her2-, Cytokeratin $5 / 6$ positive $(\mathrm{CK} 5 / 6+)$ and/or Her1+ (EGFR)). Tumors which were negative at immunohistochemical staining for all markers (ER, PR, Her2, Her1, and CK5/6) were considered unclassified subtype [4]. According to this classification, we performed immunohistochemical staining for ER, PR, Her2, Her1, CK8/18, basal CK5/6 and KI67 in paraffin sections from blocks of breast cancer. The aim of the present study was to estimate the prevalence of breast cancer subtypes in patients of the north east region of Morocco, and to correlate between clinical and pathological characteristics.

\section{Methods}

Patients this study was approved by Ethical Committee of Hassan II teaching hospital Center. A total of three hundred and ninety patients diagnosed with invasive breast carcinoma were examineted. Clinical information was retrieved from the medical records. Breast cancers were classified according to the World Health Organization (WHO, 2003) [7] while histological grading and staging were performed according to Modified Bloom-Richardson classification [8] and American Joint Committee on Cancer (AJCC) [9], respectively.

\section{Immunohistochemical study}

Tumors sections were deparaffinized and rehydrated. Peroxide blocking was done with $0.4 \% \mathrm{H} 2 \mathrm{O} 2$. Antigen retrieval was achieved by heat retrieval using a pressure cooker. After washing, the slides were treated with protein blocking agent (UltraTech HRP, Immunotech) then incubated with the following primary antibodies: antihuman ER (ER1D5, Immunotech), PR (PR10A9, Immunotech), CK5/6 (D5/16B4, Cell Marque), CK8/18 (RTU5D3, Novocastra), Ki67 (SP6 Cell Marque). After rinsing with PBS, the slides were incubated with a secondary biotinylated antibody (Immunotech). The slides were then rinsed with PBS. Sections were then incubated with streptavidin-peroxidase reagent. Staining for the slides was developed with Amino-Ethyl-Carbazole (Ultra Tech AEC, Immunotech) and then the slides were counterstained with hematoxylin, hydrated, and mounted. For Her2, immunohistochemical was carried out using with HercepTest (A0485, Dako) according to the commercial instructions for use.

Immunohistochemical surrogate biomarkers of molecular classification Immunohistochemical subtypes were defined as follows: Luminal A (ER + and/or PR+, Her2-, KI67<14\%), luminal B (ER + and/or PR+, Her2 +/-, Ki67>14\%), basal-like (ER-, PR-, Her2-, and CK5/6+, CK14+), Her2+/ER-, and unclassified subtype (negative for all markers) (Table 1). CK8/18 expression was used for confirmation the luminal subtypes.

Positive controls were included in each staining run and consisted of breast cancers known to express each of the antigens of interest. Cases were considered positive for ER and PR according to standardized guidelines using a cut-off of $\geq 1 \%$ stained tumour nuclei. Fluorescence in situ hybridization (FISH) study

All Her2 score 2+ cases were analyzed by FISH. They were performed using the PathVysion HER2 DNA Probe (Abbott Vysis Inc., Downers Grove, IL) according to the manufacturer's protocol. The probe cocktail included the LSI HER-2/neu probe and the CEP17 probe. Fluorescence signals were analyzed and digitalized using the CytoVisionTM image analysis system (Applied Imaging International Ltd., Newcastle-Upon-Tyne, UK). Between 60 and 100 nuclei were scored for each case. Signal ratios (HER2: CEP17) $\geq 2$, 2 were classified as amplified. In the absence of positive FISH data, tumors scored 2+ by IHC were considered as negative for HER-2. Her2 was scored based on a 0 to 3 scale according to the criteria set by ASCO (American Society of Clinical Oncology/College of American Pathologists) [10]. Scores 0 and $1+$ were considered as negative; score $2+$ was considered borderline; and score $3+$ was considered as strongly positive. FISH was performed on the borderline cases (score 2+).

\section{KI67}

In breast cancer, immunohistochemical assessment of the proportion of cells staining for the nuclear antigen Ki67 has become the most widely used method for comparing proliferation between tumor samples. Potential

$\begin{aligned} & \text { Table } \mathbf{1} \text { Immunohistochemical characterization of } \\
& \text { molecular subtypes of breast cancer }\end{aligned}$
\begin{tabular}{ll}
\hline Molecular subtype & Immunohistochemical characterization \\
\hline Luminal A & ER (+) et Her-2 (-) et CK8/18 (+), Ki67<14\% \\
Luminal B & ER (+) et Her-2 (+) \\
& ou ER (+), Her-2 (-) et Ki67>14\% \\
Her-2 & ER (-) et Her-2 (+) \\
Basal & ER (-) et Her-2 (-) et CK5/6 (+) \\
\hline
\end{tabular}


uses include prognosis, prediction of relative responsiveness or resistance to chemotherapy or endocrine therapy, estimation of residual risk in patients on standard therapy and as a dynamic biomarker of treatment efficacy in samples taken before, during, and after neoadjuvant therapy, particularly neoadjuvant endocrine therapy. Cases were considered positive for ki67 according to standardized guidelines using a cut-off of $\geq 14 \%$ stained tumour nuclei with moderate intensity (Figure 1).

\section{Statistical analysis}

Statistical analysis was performed in the Department of Epidemiology, of the Faculty of Medicine and Pharmacy of Fez and was carried out using Epi-Info (3.3.2).

\section{Results and discussion}

The study was achieved on 390 patients diagnosed with infiltrating breast cancer and managed at the Department of pathology in Hassan II University Hospital in Fez. The patient's average age at diagnosis was 46 years (ranging from 22 to 92 years). The tumor clinical stage on first diagnosis, according to American Joint Committee on Cancer Staging Systems, showed that 47 women $(14.5 \%)$ are at stage I, $108(33 \%)$ are at stage II, $114(35 \%)$ are at stage III and $57(17.5 \%)$ at stage IV. After histological analysis, the tumor average size was $3.7 \pm 2.6 \mathrm{~cm}$ (ranging from 0.2 to $16 \mathrm{~cm}$ ). Most of these tumors (87, 2\%) were diagnosed as invasive carcinoma (IC NOS) while
5, 4\% were invasive lobular carcinomas (ILC), $2 \%$ were metaplastic carcinoma (MC), and few patients had cancers of rare histology $(5,4 \%)$, which were summarized as "other types" in our study. The histological grade distribution for luminal B were grade II (59.9\%), grade III (30.6\%) but few patients only were grade I $(9,6 \%)$ with $\mathrm{p}<0,0001$. Vascular emboli were detected in $71.2 \%$ of patient's luminal B. The status of lymph nodes was determined for 326 patients among which $68,8 \%$ had positive lymph nodes and $12.5 \%$ had distant metastasis.

The immunohistochemical study showed that $67,1 \%$ patients were ER positives, $63,7 \%$ were PR positive, and 24, $7 \%$ were Her2 positive. Therefore, Luminal B subtype was more prevalent $(41.8 \%)$, followed by luminal A (30.5\%), basal-like (13, 6\%), Her2-overexpressing (9, 2\%), and unclassified subtype (4.9\%). The luminal B subtypes present a higher median tumor size (T2 and T3) as like basal-like and Her2-overexpressing. IHC subtypes were significantly different by histological grade $(\mathrm{p}<0.00001)$. The luminal $\mathrm{B}$ also represented a higher percentage of cases with histological grade II and III (90, 5\%) like, basal-like and Her2-overexpressing subtypes (90,2\% and $96 \%$ respectively) and a very low percentage of tumors with histological grade I $(9,6 \%, 9,8 \%$ and $4 \%$, respectively). The Luminal B subtype had 71, 2\% vascular emboli, approach Her2-overexpressing subtypes (75,6\%). In this study, the luminal B tumors had average percentage of lymph node metastasis $(68,8 \%)$ and distant metastasis

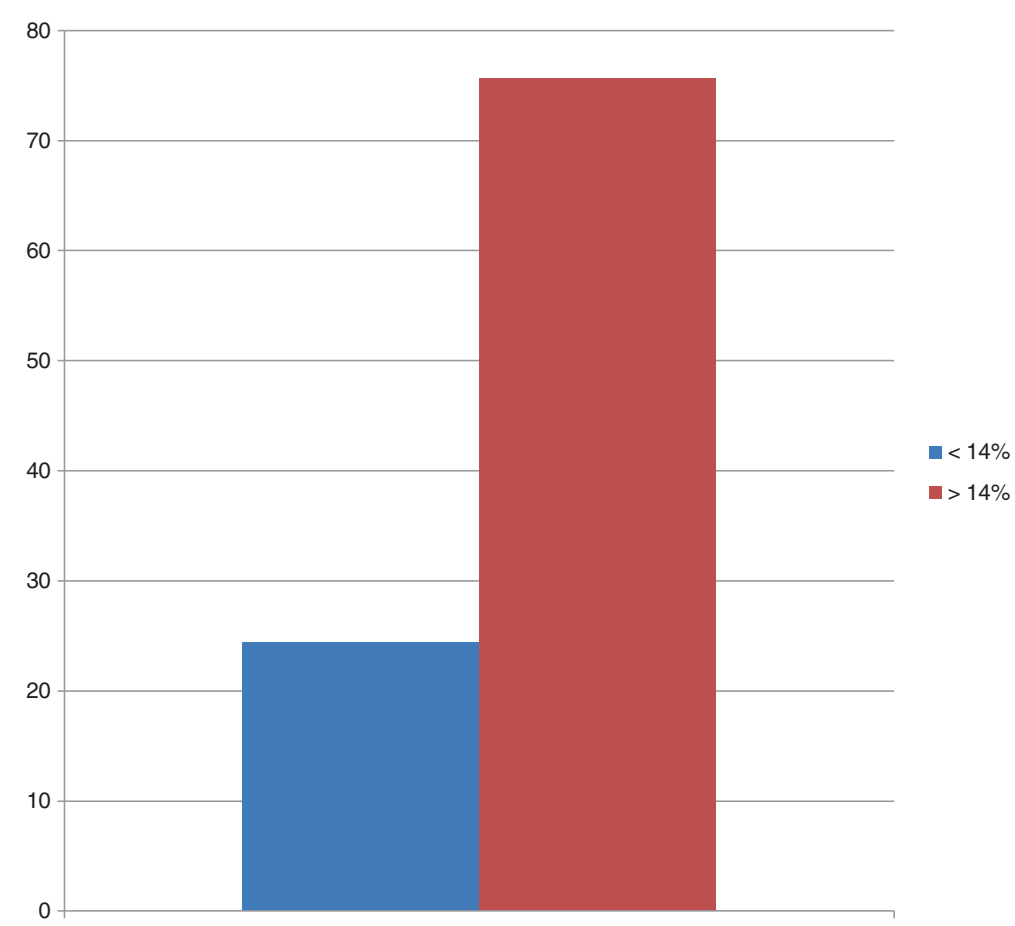

Figure 1 Distribution by the proliferation index assessed by Ki 67 . 
(12, 5\%). Table 2 Twenty-two patients (12.2\%) died because of cancer-related events during the follow-up. Among these patients, $25 \%$ belong to basal-like, 22\% belong to unclassified subtype group. $20 \%$ to Her2overexpressing group, $17 \%$ to luminal B and only $5 \%$ in luminal A.

Molecular studies of breast cancer revealed biological heterogeneity of the disease and opened new perspectives for personalized therapy. While multiple gene expression-based systems have been developed, current clinical practice is largely based upon conventional clinical and pathologic criteria. Digital image analysis (DA) with multivariate statistics of the data opens new opportunities in this field [11].

Recently, gene expression studies, using microarray technology, confirmed that the heterogeneity of clinical response could be correlated with different molecular profiles of breast cancers [1]. Arvydas and al reported a series of tissue microarrays of 109 patients with breast ductal carcinoma, were stained for a set of 10 IHC markers (ER, PR, HER2, Ki67, AR, BCL2, HIF-1 $\alpha$, SATB1, p53, and p16) [12]. This study demonstrates that factor

Table 2 Prevalence of intrinsic subtypes and clinico-pathological characteristics

\begin{tabular}{|c|c|c|c|c|c|}
\hline subtype & luminal $A$ & luminal B & Her-2 & Basallike & Unclassified \\
\hline \multicolumn{6}{|l|}{ Histological type \% } \\
\hline IDC Nos & 30,5 & 41,1 & 10,9 & 13,9 & 3,6 \\
\hline ILC & 47,6 & 42,9 & 4,8 & 0,0 & 4,8 \\
\hline Other types & 25 & 8,3 & 16,7 & 50 & 0,0 \\
\hline \multicolumn{6}{|l|}{$P<0,00001$} \\
\hline \multicolumn{6}{|l|}{ Histological grade\% } \\
\hline 1 & 28,3 & 9,6 & 9,8 & 4 & 15,8 \\
\hline II & 59,2 & 59,9 & 56,1 & 32 & 26,3 \\
\hline III & 12,5 & 30,6 & 34,1 & 64 & 57,9 \\
\hline \multicolumn{6}{|l|}{$P<0,0001$} \\
\hline \multicolumn{6}{|l|}{ Vascular emboli \% } \\
\hline Positive & 60,8 & 71,2 & 75,6 & 64,4 & 63,2 \\
\hline negative & 39,2 & 28,8 & 24,4 & 35,3 & 36,8 \\
\hline \multicolumn{6}{|l|}{$p=0,29$} \\
\hline \multicolumn{6}{|l|}{ Intraductal carcinoma \% } \\
\hline Yes & 55 & 39,5 & 41,5 & 25,5 & 78,9 \\
\hline No & 45 & 60,5 & 58,5 & 74,5 & 21,1 \\
\hline \multicolumn{6}{|l|}{$P=0,0013$} \\
\hline \multicolumn{6}{|l|}{ Tumour size $(\mathrm{cm})$} \\
\hline $\mathrm{T} 1$ & 42,9 & 31,8 & 9,1 & 12,5 & 25 \\
\hline $\mathrm{T} 2$ & 34,5 & 44,76 & 45,5 & 46,9 & 25 \\
\hline T3 & 14,3 & 18,8 & 27,3 & 15,6 & 12,5 \\
\hline T4 & 8,4 & 4,7 & 18,2 & 25 & 37,5 \\
\hline \multicolumn{6}{|l|}{$P=0,0003$} \\
\hline \multicolumn{6}{|l|}{ Necrosis \% } \\
\hline Present & 8,5 & 17,8 & 22,2 & 22,6 & 10,5 \\
\hline Absent & 91,5 & 82,8 & 77,8 & 77,4 & 89,5 \\
\hline \multicolumn{6}{|l|}{$P=0,0698$} \\
\hline Positive Lymph nodes \% & 52,4 & 68,8 & 86,7 & 81,3 & - \\
\hline \multicolumn{6}{|l|}{$P=0,0066$} \\
\hline \multicolumn{6}{|l|}{ Distant metastasis \% } \\
\hline Yes & 4,5 & 12,5 & 17,9 & 26,7 & 28,6 \\
\hline No & 95,5 & 87,5 & 82,1 & 73,3 & 71,4 \\
\hline$P=0,0030$ & & & & & \\
\hline
\end{tabular}


analysis of multiple IHC biomarkers measured by automated DA is an efficient exploratory tool clarifying complex interdependencies in the breast ductal carcinoma IHC profiles. Major factor of the aggressive disease behaviour (i-Grade) is characterized by opposite loadings of ER/PR/AR/BCL2 and Ki67/HIF-1 $\alpha$. The i-Grade factor scores represent integral quantitative characteristics that reveal bimodal distribution and are strongly associated with the histological grade and relevant intrinsic subtypes. In HR-positive tumours, the aggressiveness of the tumour is best reflected by the combination of Ki67 and ER, rather than Ki67 and BCL2. Inverse relation between HER2 and PR expression in the HR-positive tumours which, along with the inverse relation between Ki67 and ER, may shed the light into the differential information conveyed by the ER and PR expression. Remarkably, SATB1 along with HIF- $1 \alpha$ reflected the second major factor of variation in patients with breast cancer; furthermore, in the HR-positive group they were inversely associated with the HR and BCL2 expression and represented the major factor contributing to the variation in the IHC data set. However, this factor was not associated with the clinicopathologic categories studied. Biological meaning of this variation remains unclear: HIF- $1 \alpha$ and SATB1 may convey important biological messages other than the aggressiveness of the disease reflected by Ki67 expression and histological grade. Meanwhile, the authors, support the notion that the suggested prognostic significance of SATB1 may be related to its inverse relation to the ER expression. Finally, this analysis confirms high expression levels of p16 in Triple-negative tumours [12]. In our study, based on recent updated IHC subtype definition by Prat et al. [4], we estimated the prevalence of breast cancer subtypes in patients from the north east Moroccan region and established the correlations between clinico-pathological characteristics. The patients recruited in our university hospital were younger than in western series; the average age at diagnosis was 46.8 years. In terms of clinical staging, only $14.5 \%$ patients were diagnosed at stage I, while $33 \%$ were at stage II, $35 \%$ at stage III and $17.5 \%$ at stage IV. On the other hand, after histological analysis the average tumor size was $3.7 \mathrm{~cm}$ and $75 \%$ of cases measured more than $2 \mathrm{~cm}$. A majority of tumors were stage II or III. Our data showed that more than $50 \%$ of patients of all subtypes presented positive lymph nodes and more than $12 \%$ of cases had distant metastasis on first diagnosis. This could be due to late consultation during the progression of the disease in our region as well as to lack of the Medicare coverage, lack of screening mammography program and women's awareness trainings particularly in rural area. The predominant histology type in this study was invasive cancer (87.4\%), similar to most breast cancer studies worldwide.
Overexpression of the protein and/or amplification of the HER2 gene have been reported in approximately 20 to $30 \%$ of breast cancers, similar to what was found in our patients $(24,7 \%)$. Her2+ tumors are associated with either poor prognosis or with response to trastuzumab [13]. Our results showed a distribution of breast cancer subtypes non similar to what was reported by other immunohistochemical studies [4]. In this study, the prevalence of luminal B $(41,8 \%)$ and is the more frequent subtype. Considering the fact that all patients came from a university hospital the possible effects of a selection bias should be regarded. Cancer registry available includes only data from the university hospital and not all North African population of Morocco. Triplenegative subtype represented approximately $13,6 \%$ of our series and $78 \%$ of them were basal-like tumors. These results were similar to what was found in other studies $[4,14]$. The basal-like group was defined by immunohistochemistry, as being negative for ER, PR, and Her2 and positive for Ck5/6, CK14 and/or Her1 (EGFR). It is important to study whether EGFR is overexpressed in patients with breast cancer since these patients can be given specific EGFR molecule tyrosine kinase inhibitors such as gefitinib and lapatinib $[15,16]$. There are only a few reports regarding the overexpression of EGFR, with these studies indicating $8-36 \%$ of breast cancers over express this protein. However, systematic studies appraising EGFR gene amplification and mutations in the same set of cases among Chinese female patients with breast cancer are absent [17-19]. EGFR gene mutations are infrequent in breast cancers. This suggested that EGFR mutation analysis is not useful as a screening test for sensitivity to anti-EGFR therapy for breast cancers [20]. In literature, the luminal B tumors are associated with poor recurrence-free and disease-specific survivals in all adjuvant systemic treatment categories including hormone therapy; the identification of specific signaling pathways driving luminal B biology is paramount to improve treatment. Sircoulomb et al. and Holland et al. have independently identified the ZNF703 gene, located in chromosomal region 8p12, as preferentially amplified in luminal B tumours [21].

The natural history of breast cancer involves progression through defined pathological and clinical stages, starting with ductal hyperproliferation, with subsequent evolution into in situ and invasive carcinomas, and finally into metastatic disease. The majority of invasive breast cancer develop over long periods of time from certain pre-existing benign lesions. The best characterized premalignant lesions recognized today are referred to as atypical ductal hyperplasia (ADH), atypical lobular hyperplasia (ALH), ductal carcinoma in situ (DCIS) and lobular carcinoma in situ (LCIS). Douglas and al, [22] reported that Lobular neoplasia (LN) and invasive 
lobular carcinoma (ILC) are detected together frequently in the same specimen and location of the tumor--in up to $90 \%$ of cases of ILC [23]. Invasive carcinomas were present with $\mathrm{LN}$ in $45.6 \%$ of cases, with similar rates of association with invasive carcinoma and ILC (47.2\%). Bratthauer and Tavassoli stratified the LNs as "lobular intraepithelial neoplasias" (LIN) and evaluated the frequency of association between LIN subtypes (1,2, and 3) and invasive carcinoma. The percentage of LIN 1 (equivalent to ALH) that was associated with invasive carcinoma was $14 \%$, and $89 \%$ of these tumors were IDCs. In the patients with LIN 3 (equivalent to LCIS), the frequency of association with IDC and ILC was 23\% and $86 \%$, respectively. The authors concluded that the advance from LIN 1 to LIN 3 was linked to a $64 \%$ increase in the frequency of invasive carcinoma and a greater than $70 \%$ rise in the likelihood of ILC [24]. Apoptosis is a frequent phenomenon in breast cancer and it can be detected by light microscopy in conventional histopathological sections or by special staining techniques. Balance between expression status of antiapoptotic and pro-apoptotic proteins determines cells to be alive or not. The key event of apoptosis occurrence is cascade activation of caspases, and inhibitor of apoptosis proteins (IAPs) play a important role in caspase inhibition. XIAP is the most potent caspase inhibitor and Smac is one of the antagonists of XIAP. Down regulation of XIAP expression or applying exogenous Smac mimics can sensitize tumor cells, especially for breast cancer cells, to chemotherapeutics and promote apoptosis $[25,26]$. In immunohistochemistry, XIAP and Smac were positive in cytoplasm of tumor cells with strong or moderate intensity, respectively. The positive ratio of XIAP $(84.3 \%, 86 / 102)$ was more higher than that of Smac $(33.3 \%, 34 / 102)$, and immunoscore of XIAP was higher than Smac in IDC too $(P<0.0001)$. It was noteworthy that 44 IDC samples were nuclear positive for XIAP, but none was for Smac. And cytoplasm positive status of XIAP nuclear positive group was stronger than the negative group $(P=0.030,0.047)$. Otherwise, Smac immunoscore was prevalent in HER2 positive group than negative group $(P<0.0001)$. Remaining data revealed that the expression status of XIAP/Smac was not correlated with patient age, tumor size, lymph node status, histologic grading, expression of ER and PR. XIAP was a potent protein for apoptosis inhibition and Smac was an important negative regulator of the former. Disturbed balance of expression between XIAP and Smac probably contributed to carcinogenesis and XIAP positive nuclear labeling was a sign of unfavourable prognosis in breast invasive ductal carcinoma [27]. Others studies have demonstrated that claudin- 6 functions as a cancer suppressor in human MCF-7 breast cancer cells. The growth inhibitory effect could be attributed to inhibition of cell proliferation and induction of apoptosis. Claudins (CLDNs) constitute a family of integral membrane proteins and have been identified as prominent structural components of tight junction ( TJ ) strands [28,29]. TJs are junctional complexes which mediate cell-to-cell adhesion in epithelial and endothelial cellular sheets [30], and which affect cell polarity and tight junction formation [28]. Guo and al reported that the apoptosis signal-regulating kinase 1 (ASK1) expression is low in breast cancer, and the levels of ASK1 mRNA and protein expression are correlated with that of claudin- 6 . They have identified a novel mechanism responsible for the pro-apoptosis function of claudin-6, and ASK1 may become a target for breast cancer treatments. The integrins, a family of transmembrane glycoproteins, play a major role in invasive and metastatic processes. Integrins are involved in cell adhesion in both cell-extracellular matrix and cell-cell interactions, and particularly, beta 1 integrin is involved in proliferation and differentiation of cells in the development of epithelial tissues. The putative role of beta 1 integrin expression on survival and metastasis in patients with breast invasive ductal carcinoma (IDC) was studied by Santos and al [31]. His study showed that beta1 integrin can be a marker of poor prognosis in breast cancer. Beta 1 integrin was overexpressed in $32.8 \%$ of IDC and was related with HER-2 and VEGF expression, and it had a significant relationship with metastasis and death , survival analysis showed that the overexpression of this protein is very significant in specific survival (number of months between diagnosis and death caused by the disease). Immunohistochemistrey is important to determinate the tumors of unknown origin (5-15\%) and specially breast cancer metastasis (1.5\%). Mammaglobin (MAG) antibody in the immunohistochemical panel for the detection of tumors of unknown origin contributed to the detection of metastasis of breast cancer. The diagnostic strategy with the highest positive predictive value (88\%) included hormone receptors and mammaglobin in serial manner [32].

\section{Conclusions}

We have shown that simple IHC-based classification of breast tumors can be helpful. Since the predictive power of IHC criteria appears to be similar to that of gene expression analysis, this information can be used to improve therapeutic decisions, mainly for luminal B, Her2- overexpressing and basal-like subtypes. The luminal B subtype was associated with a poor prognosis and unfavorable clinico-pathological characteristics. In addition, findings concerning tumors stages are alarming and highlight the importance of early screening and the urgent need to improve women's awareness of breast cancer in our region. Our results should be confirmed 
by large studies to be conducted in other institutions and hospitals including patients coming from different regions of Morocco.

\author{
Abbreviations \\ HER2: Epithelial human receptor 2; ER: Estrogen receptor; PR: Progesterone \\ receptor; IHC: Immunohistochemistry; CK: Cytokeratin; WHO: World Health \\ Organization; AJCC: American Joint Committee on Cancer; \\ FISH: Fluorescence in situ hybridization; IC NOS: Invasive carcinoma not \\ otherwise specified; ILC: Invasive lobular carcinoma; MC: Metaplastic \\ carcinoma; DA: Digital image analysis; EGFR: Epidermal growth factor \\ receptor; ADH: Atypical ductal hyperplasia; ALH: Atypical lobular hyperplasia; \\ DCIS: Ductal carcinoma in situ; LCIS: Lobular carcinoma in situ; LN: Lobular \\ neoplasia; LIN: Lobular intraepithelial neoplasia; ASK1: Apoptosis signal- \\ regulating kinase 1.
}

\section{Competing interests}

The authors declare that they have no competing interests.

\section{Authors' contributions}

All authors analyzed, interpreted and approved the final manuscript

\section{Funding}

This study received no specific grant from any funding agency in the public, commercial or not-for-profit sectors

\section{Author details}

'Department of Pathology, Hassan II teaching hospital, Fez, Morocco. ${ }^{2}$ Department of Gynecology, Hassan II teaching hospital, Fez, Morocco. ${ }^{3}$ Department of Oncology, Hassan II teaching hospital, Fez, Morocco.

Received: 15 October 2012 Accepted: 4 December 2012

Published: 7 December 2012

\section{References}

1. Perou CM, Sorlie T, Eisen MB, et al: Molecular portraits of human breast tumours. Nature 2000, 406:747-752.

2. Sorlie T, Perou CM, Tibshirani R, et al: Gene expression patterns of breast carcinomas distinguish tumor subclasses with clinical implications. Proc Natl Acad Sci USA 2001, 98:10869-10874.

3. Sorlie T: Molecular portraits of breast cancer: tumour subtypes as distinct disease entities. Eur J Cancer 2004, 40:2667-26675.

4. Aleix P, Parker JS, Olga K, Cheng F, Chad L, Herschkowitz JI, Xiaping H, Perou CM: Phenotypic and molecular characterization of the claudin-low intrinsic subtype of breast cancer. Breast Cancer Res 2010, 12:R68

5. Bhargava R, Striebel J, Beriwal S, et al: Prevalence, morphologic features and proliferation indices of breast carcinoma molecular classes using immunohistochemical surrogate markers. Int I Clin Exp Pathol 2009, 2:444-455.

6. Tang P, Wang J, Bourne P: Molecular classifications of breast carcinoma with similar terminology and different definitions: are they the same? Hum Pathol 2008, 39:506-513.

7. Tavassoli F, Devilee P (Eds): Pathology and genetics of tumours of the breast and female genital organs, world health organization classification of tumours. Lyon: IARC Press; 2003.

8. Elston CW, Ellis IO: Pathological prognostic factors in breast cancer. I. The value of histological grade in breast cancer: experience from a large study with long-term follow up. Histopathology 1991, 19(5):403-410.

9. American Joint Committee on Cancer: AJCC cancer staging handbook; 2010. www.springer.com/medicine/surgery/cancer+staging?SGWID=0-40654-0-0-0, Last accessed on 14/1/2011.

10. Wolff AC, Elizabeth $M$, Hammond $H$, et al: Guideline recommendations for human epidermal growth factor receptor 2 testing in breast cancer. American Society of Clinical Oncology/College of American Pathologists. J Clin Oncol 2007, 25:118-145.

11. Prat A, Ellis MJ, Perou CM: Practical implications of gene-expression-based assays for breast oncologists. Nat Rev Clin Oncol 2011, 9(1):48-57.

12. Arvydas L, Aida L, Valerijus O, Darius D, Sonata J, Juozas L: Immunohistochemistry profiles of breast ductal carcinoma: factor analysis of digital image analysis data. Diagn Pathol 2012, 7:27.
13. Ross JS, Fletcher JA, Bloom KJ, et al: Targeted therapy in breast cancer. Mol Cell Proteomics 2004, 3:379-398.

14. Junichi $\mathrm{K}$, Takoya M, Takanori I, et al: The prevalence of intrinsic subtypes and prognosis in breast cancer patients of different races [abstract] Breast 2007, 16(Suppl 2):S72-S77.

15. Ciardiello F, Troiani T, Caputo F, De Laurentiis M, Tortora G, Palmieri G, De Vita F, Diadema MR, Orditura M, Colantuoni G, et al: Phase II study of gefitinib in combination with docetaxel as first-line therapy in metastatic breast cancer. Br J Cancer 2006, 94:1604-1609.

16. Baselga J, Albanell J, Ruiz A, Lluch A, Gascon P, Guillem V, Gonzalez S, Sauleda S, Marimon I, Tabernero JM, et al: Phase II and tumor pharmacodynamic study of gefitinib in patients with advanced breast cancer. J Clin Oncol 2005, 23:5323-5333.

17. Tsutsui S, Kataoka A, Ohno S, Murakami S, Kinoshita J, Hachitanda Y: Prognostic and predictive value of epidermal growth factor receptor in recurrent breast cancer. Clin Cancer Res 2002, 8:3454-3460.

18. Tsuda H, Morita D, Kimura M, Shinto E, Ohtsuka Y, Matsubara O, Inazawa J, Tamaki K, Mochizuki H, Tamai S, Hiraide H: Correlation of KIT and EGFR overexpression with invasive ductal breast carcinoma of the solidtubular subtype, nuclear grade 3 , and mesenchymal or myoepithelial differentiation. Cancer Sci 2005, 96:48-53.

19. Walker RA, Dearing SJ: Expression of epidermal growth factor receptor mRNA and protein in primary breast carcinomas. Breast Cancer Res Treat 1999, 53:167-176.

20. Kobayashi S, Boggon TJ, Dayaram T, Janne PA, Kocher O, Meyerson M, Johnson BE, Eck MJ, Tenen DG, Halmos B: EGFR mutation and resistance of non-small-cell lung cancer to gefitinib. N Engl J Med 2005, 352:786-792.

21. Kristensen VN: Divide and conquer: the genetic basis of molecular sub classification of breast cancer. EMBO Mol Med 2011, 3:183-185.

22. Gomes DS, Débora B, Porto SS, Helenice G: Lobular neoplasia: frequency and association with other breast lesions. Diagn Pathol 2011, 6:74. 9 August 2011.

23. Abdel-Fatah TM, Powe DG, Hodi Z, Lee AH, Reis-Filho JS, Ellis IO: High frequency of coexistence of columnar cell lesions, lobular neoplasia, and low grade ductal carcinoma in situ with invasive tubular carcinoma and invasive lobular carcinoma. Am J Surg Pathol 2007, 31:417-426.

24. Bratthauer GL, Tavassoli FA: Lobular intraepithelial neoplasia: previously unexplored aspects assessed in 775 cases and their clinical implications. Virchows Arch 2002, 440:134-138.

25. Peng XH, Karna P, O'Regan RM, Liu X, Naithani R, Moriarty RM, Wood WC, Lee HY, Yang L: Down-regulation of inhibitor of apoptosis proteins by deguelin selectively induces apoptosis in breast cancer cells. Mol Pharmacol 2007, 71(1):101-111.

26. Bockbrader KM, Tan M, Sun Y: A small molecule Smac-mimic compound induces apoptosis and sensitizes TRAIL- and etoposide-induced apoptosis in breast cancer cells. Oncogene 2005, 24(49):7381-7388.

27. Yutao Z, Jianhua Z, Yun T, Feng L, Hongyuan Z, Bofang P, Chifeng Z, Rong $F$ : X-linked inhibitor of apoptosis positive nuclear labeling: a new independent prognostic biomarker of breast invasive ductal carcinoma. Diagn Pathol 2011, 6:49.

28. Matter K, Balda MS: Signaling to and from tight junctions. Nat Rev Mol Cell Biol 2003, 4:225-236.

29. Tsukita S, Furuse M: Occludin and claudins in tight-junction strands: leading or supporting players? Trends Cell Biol 1999, 9:268-273.

30. Tsukita S, Furuse M, Itoh M: Multifunctional strands in tight junctions. Nat Rev Mol Cell Biol 2001, 2:285-293.

31. Santos PB, Zanetti JS, Silva AR, Beltrão EIC: Beta 1 integrin predicts survival in breast cancer: a clinicopathological and immunohistochemical study. Diagnostic Pathology 2012, 7:104.

32. MariadelasMercedes N, Fernando P, Florencia P, Néstor L, Hugo K, Silvana N, Alejandro G, Alejandra A, Boris E, Valeria D, Miembro de la Carrera de Investigador del Consejo Nacional de Investigaciones Científicas y Técnicas (CONICET): Immunohistochemical characterization of neoplastic cells of breast origin. Diagn Pathol 2012, 7:73.

doi:10.1186/1746-1596-7-170

Cite this article as: El Fatemi et al:: Luminal B tumors are the most frequent molecular subtype in breast cancer of North African women: an immunohistochemical profile study from Morocco. Diagnostic Pathology 2012 7:170 